\title{
Mechanisms of acquired resistance to fibroblast growth factor receptor targeted therapy
}

\author{
David K. Lau ${ }^{1,2}$, Laura Jenkins ${ }^{1,2}$, Andrew Weickhardt ${ }^{1,2,3}$ \\ 'Olivia Newton John Cancer Research Institute, Heidelberg, Victoria 3084, Australia. \\ ${ }^{2}$ School of Cancer Medicine, La Trobe University, Heidelberg, Victoria 3084, Australia. \\ ${ }^{3}$ Department of Medical Oncology, Austin Health, Heidelberg, Victoria 3084, Australia.
}

Correspondence to: A/Prof. Andrew Weickhardt, Medical Oncologist/Translational research fellow, Olivia Newton-John Cancer Research Institute, 145 Studley Road, Heidelberg, Victoria 3084, Australia. E-mail: andrew.weickhardt@onjcri.org.au

\begin{abstract}
How to cite this article: Lau DK, Jenkins L, Weickhardt A. Mechanisms of acquired resistance to fibroblast growth factor receptor targeted therapy. Cancer Drug Resist 2019;2:568-79. http://dx.doi.org/10.20517/cdr.2019.42
\end{abstract}

Received: 17 May 2019 First Decision: 18 Jun 2019 Revised: 4 Jul 2019 Accepted: 10 Jul 2019 Published: 19 Sep 2019

Science Editor: Helen M. Coley Copy Editor: Cai-Hong Wang Production Editor: Jing Yu

\begin{abstract}
Oncogenic activation of the fibroblast growth factor receptor (FGFR) through mutations and fusions of the FGFR gene occur in a variety of different malignancies such as urothelial carcinoma and cholangiocarcinoma. Inhibition of the kinase domain of the FGFR with targeted oral FGFR inhibitors has been shown in both preclinical and early phase clinical trials to lead to meaningful reductions in tumour size and larger confirmatory randomized trials are underway. Acquired resistance to FGFR inhibition using a variety of mechanisms that includes, activation of alternate signaling pathways and expansion of tumour clones with gatekeeper mutations in the FGFRgene. This review summarizes the acquired resistance mechanisms to FGFR therapy and therapeutic approaches to circumventing resistance.
\end{abstract}

Keywords: Fibroblast growth factor receptor, targeted therapy, acquired resistance

\section{INTRODUCTION}

The fibroblast growth factor receptor (FGFR) is a membrane bound protein that regulates cellular functions including cell proliferation, cell survival, differentiation and migration ${ }^{[1]}$. Activation of the FGFR family (FGFR1, FGFR2, FGFR3 and FGFR4) leads to increased downstream activation of oncogenic pathways such as MAPK and $\mathrm{AKT}^{[2]}$. Amplifications, mutations and aberrant fusions of the FGFR gene lead to constitutively activated downstream signaling of these pathways with enhanced cellular growth and migration $^{[3]}$.

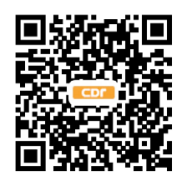


Cancers such as breast, lung, gastric, urothelial and intrahepatic cholangiocarcinoma harbor hyperactivation of FGFR signaling pathways due to oncogenic aberrations of FGFR family members, although the nature of the oncogenic alteration is different between each cancer type.

Recently, a wide variety of orally available targeted pan-FGFR inhibitors such as derazantinib (ARQ-087, Arqule) $^{[4]}$, AZD4547 (AstraZeneca) $)^{[5]}$, infigratinib (BGJ398, Novartis) ${ }^{[6]}$, erdafitinib (JNJ-42756493, Janssen) ${ }^{[7]}$, TAS-120 (Taiho) ${ }^{[8]}$ and pemigatinib (INCB054828, InCyte) ${ }^{[9]}$ with specificity for inhibition of the kinase domain of the activated FGFR protein have evolved from preclinical testing to early phase clinical trials. Anti-tumour activity in clinical trials of urothelial carcinoma and cholangiocarcinoma has led to larger confirmatory clinical trials that may lead to registration of these agents.

Preclinical trials and early phase testing have demonstrated resistance occurs to these targeted agents and similar phenomenon is seen with other kinase inhibitors, such as $\mathrm{EGFR}^{[10]}, \operatorname{ROS}^{[11]}$ and $\mathrm{CKIT}$ inhibitors ${ }^{[12]}$. The goal of this review is to outline the outcomes of preclinical and clinical studies of acquired resistance to selective FGFR inhibition and development of rational therapeutic strategies to circumvent resistance.

\section{ACTIVATING FGFR ALTERATIONS IN CANCER}

Identification of FGFR gene fusions, mutations and amplifications in a range of cancer types has driven the study of FGFR inhibitors in these tissue and biomarker selected populations. Gene fusions involving FGFR2 occur in $7 \%-14 \%$ of intrahepatic cholangiocarcinomas. The first reported constitutively active fusion gene was the FGFR2-BICC $1^{[13]}$. Other reported fusion partners of FGFR2 include CCDC6 ${ }^{[14]}, P P H L N 1^{[15]}$, AHCYL $1^{[16]}$, TXLNA1 and KCTD1 ${ }^{[17]}$. The breakpoint in nearly all FGFR2 fusions is located in exon 18, distal to the kinase domain of the FGFR receptor. In non-muscle invasive bladder cancer, FGFR3 mutations can be detected in 50\%-60\% cases. By contrast, the prevalence of FGFR 3 mutations is significantly lower in muscle invasive bladder cancer $(10 \%-15 \%)^{[18,19]}$. In addition, FGFR3 fusion rearrangements are present in $6 \%$ of muscle invasive bladder cancer ${ }^{[20]}$. FGFR2 mutations are present in $\sim 10 \%$ of endometrial cancers ${ }^{[21]}$. The results of early phase trials of FGFR inhibitors in urothelial carcinoma (FGFR3 mutations/translocations) and cholangiocarcinoma (FGFR2 fusions) are shown in Table 1.

FGFR1 amplifications are present in several tumour types including squamous non-small cell lung (10\%$20 \%)^{[22,23]}$, hormone positive breast (10\%), head and neck (10\%-17\%), squamous oesophageal (20\%) and ovarian cancer $(9 \%)^{[24]}$. FGFR2 amplifications can be detected in triple negative breast $(4 \%)^{[25]}$ and gastric cancer $(4 \%-7 \%)^{[26]}$. Low frequency FGFR alterations have been observed in sarcomas, glioma, pancreatic, renal, colorectal neuroendocrine cancers ${ }^{[24]}$. Despite strong preclinical rationale ${ }^{[27-29]}$, FGFR inhibitors in molecularly selected subgroups of squamous cell lung cancer ${ }^{[30]}$, breast cancer ${ }^{[31]}$ and gastric carcinoma ${ }^{[32]}$ to date have not shown significant efficacy, potentially due to the inclusion of tumour types defined by FGFR amplification, rather than mutations or fusions, which are more likely to respond to FGFR inhibition. The clinical evidence for targeting these tumours types and its respective FGFR genetic aberration are summarised in this section.

\section{CLINICAL TRIALS IN CHOLANGIOCARCINOMA}

In a proof of concept phase II study of infigratinib in advanced or metastatic cholangiocarcinoma with FGFR2 aberrations $(n=61)$, the overall response rate (ORR) was $14.8 \%$. Responses were observed only in patients with FGFR2 fusions $(n=48)$ where the ORR was $18.8 \%$. The median overall survival (OS) was 5.8 months (95\%CI: 4.3-7.6 months) ${ }^{[33]}$. Similar results were observed with the FGFR inhibitor derazantinib (ARQ-087). In a phase I/II open label study in patients with FGFR2 fusion cholangiocarcinoma $(n=29)$, the ORR was $20.7 \%$ and disease control rate (DCR) was $82.8 \%$. Estimated median progression free survival was 5.7 months (95\%CI: 4.04-9.2 months $)^{[34]}$. An expanded cohort using the $300 \mathrm{mg}$ dose is currently 
Table 1. Key clinical studies of selective pan-FGFR inhibitors in advanced cholangiocarcinoma and urothelial carcinoma

\begin{tabular}{|c|c|c|c|c|c|}
\hline Study (author/year) & Drug & Phase, patients & $\begin{array}{c}\text { Median PFS } \\
(95 \% \mathrm{CI})\end{array}$ & $\begin{array}{l}\text { Median OS } \\
(95 \% \mathrm{CI})\end{array}$ & ORR \\
\hline \multicolumn{6}{|l|}{ Cholangiocarcinoma } \\
\hline Javle et al.. ${ }^{[33]}, 2018$ & Infigratinib & $\begin{array}{l}\text { Phase II single arm } \\
\text { FGFR2 fusion }(78.7 \%) n=67\end{array}$ & $\begin{array}{l}5.8 \text { months } \\
(4.3-7.6)\end{array}$ & NR & $14.8 \%(9 / 61)$ \\
\hline $\begin{array}{l}\text { Mazzaferro et al. }{ }^{[34]}, \\
2019\end{array}$ & Derazantinib & $\begin{array}{l}\text { Phase II single arm } \\
\text { FGFR2 fusion } n=29\end{array}$ & $\begin{array}{l}5.7 \text { months } \\
(4.04-9.2)\end{array}$ & NR & $20.7 \%(6 / 29)$ \\
\hline $\begin{array}{l}\text { Meric-Bernstam et al. }{ }^{[37]}, \\
2018\end{array}$ & TAS-120 & $\begin{array}{l}\text { Phase I dose escalation } \\
\text { FGFR2 fusions } n=28 \\
\text { Other FGFR alterations }\end{array}$ & NR & NR & $\begin{array}{l}\text { FGFR2 fusions } 25 \% \\
(7 / 28) \\
\text { Other FGFR } \\
\text { alterations } 18 \%(3 / 17)\end{array}$ \\
\hline $\begin{array}{l}\text { Hollebecque et al. }{ }^{[9]}, \\
2018\end{array}$ & Pemigatinib & $\begin{array}{l}\text { Phase II } \\
\text { Cohort A: FGFR2 fusion }(n=47) \\
\text { Cohort B: FGFR/FGF alterations } \\
(n=22) \\
\text { Cohort C: no FGFR alteration } n=18\end{array}$ & $\begin{array}{l}\text { A: } 6.8 \\
\text { months (3.6- } \\
9.2) \\
\text { B: } 1.5 \text { months } \\
\text { C: } 1.4 \text { months }\end{array}$ & NR & $\begin{array}{l}\text { A: } 18 \%(8 / 45) \\
\text { B: } 0 \%(0 / 22) \\
\text { C: } 0 \%(0 / 18)\end{array}$ \\
\hline Chen et al. ${ }^{[36]}, 2018$ & Erdafitinib & $\begin{array}{l}\text { Asian cohort } \\
\text { FGFR2 fusions/ mutations } n=12\end{array}$ & $N R$ & NR & $45.5 \%(5 / 11)$ \\
\hline \multicolumn{6}{|l|}{ Urothelial carcinoma } \\
\hline Pal et al. ${ }^{[39]}, 2018$ & Infigratinib & $\begin{array}{l}\text { Phase II single arm } \\
\text { FGFR3 fusion } n=67\end{array}$ & $\begin{array}{l}3.75 \text { months } \\
(3.09-5.39)\end{array}$ & $\begin{array}{l}7.75 \text { months } \\
(5.65-11.60)\end{array}$ & $25.4 \%(17 / 67)$ \\
\hline Loriot et al. ${ }^{[38]}, 2018$ & Erdafitinib & $\begin{array}{l}\text { Phase II, single arm } \\
\text { FGFR2/FGFR3 fusions/mutations } \\
n=96\end{array}$ & 5.5 months & 13.8 months & $40 \%$ \\
\hline Joerger et al. ${ }^{[86]}, 2018$ & Rogaratinib & $\begin{array}{l}\text { Phase I expansion } \\
\text { FGFR3 mRNA alterations/high } \\
\text { expression }(n=52)\end{array}$ & NR & NR & $24 \%(12 / 51)$ \\
\hline Necchi et al. ${ }^{[87]}, 2018$ & Pemigatinib & $\begin{array}{l}\text { Phase II } \\
\text { Cohort A: FGFR3 mutations/ } \\
\text { fusions }(n=64) \\
\text { Cohort B: Other FGFR/FGF } \\
\text { alterations }(n=36)\end{array}$ & NR & NR & $\begin{array}{l}\text { A: } 25 \%(13 / 51) \\
B: 3 \%(1 / 36)\end{array}$ \\
\hline
\end{tabular}

PFS: progression free survival; OS: overall survival; $\mathrm{Cl}$ : confidence interval; ORR: objective response rate; NR: not reported

underway (NCT03230318). In a phase I dose expansion cohort of FGFR2 translocated cholangiocarcinoma receiving erdafinitib $(n=11)$, objective responses were observed in 3 patients $(27.3 \%)$ with an additional 3 patients achieving stable disease ${ }^{[35]}$. Preliminary results of erdafitinib in a phase II study in an Asian cohort reported an ORR of $45 \%{ }^{[36]}$. Clinical activity has been reported in non-randomised studies of FGFR2 translocated cholangiocarcinoma with TAS-120 and pemigatinib ${ }^{[0,37]}$. A phase III study comparing infigratinib and chemotherapy (cisplatin and gemcitabine) in FGFR2 fusion cholangiocarcinoma in the first-line setting is currently underway (NCT03773302).

\section{CLINICAL TRIALS IN UROTHELIAL CARCINOMA}

In the BLC2001 phase II study of metastatic urothelial carcinoma with FGFR alterations, 96 patients who received at least one line of chemotherapy or were chemotherapy naïve and ineligible to receive cisplatin were enrolled to receive erdafitinib $8 \mathrm{mg} / \mathrm{d}$ in 28 -day cycles with doses increased to $9 \mathrm{mg} / \mathrm{d}$ based on serum phosphorus (phosphate) levels. The ORR was $42 \%$ (3\% complete response, $39 \%$ partial response). The progression free survival (PFS) and OS were 5.5 and 13.8 months respectively ${ }^{[38]}$. Based upon these results, erdafitinib was granted breakthrough therapy designation by the Food and Drug Administration. In a phase II trial, among 67 patients with FGFR3 mutations/fusion positive bladder cancer enrolled to receive infigratinib, objective responses were observed in $25.4 \%$ of subjects. However, responses were not sustained with median PFS of 3.75 months (95\%CI: 3.09-5.39 months) ${ }^{[39]}$. A randomised phase III trial comparing the efficacy of erdafitinib against chemotherapy or pembrolizumab is underway (NCT03390504). A trial in the adjuvant setting with pemigatinib is also recruiting (NCT03656536). 


\section{CLINICAL TRIALS IN GASTRIC CANCER}

In contrast to the trials of FGFR inhibitors in cholangiocarcinoma and urothelial cancer, results from gastric cancer studies have been disappointing. Despite preclinical evidence of FGFR amplification corresponding with sensitivity to this drug class, results from treating patients with tumours harbouring FGFR amplification have been disappointing. In a phase I expanded cohort study of AZD4547, in 13 patients with FGFR amplified gastric cancer one patient had a partial response. Of note, the partial responder had clusters of FGFR1/2 gene amplification ${ }^{[40]}$. In a phase II translational clinical trial, 3 of the 9 patients with FGFR2 amplified GC achieved an objective response to AZD4547 patients with homogenous, high level FGFR2 amplification (FISH ratio $>5$ ) were most likely to respond ${ }^{[41]}$. In a phase I study of LY2874455 in patients with advanced-stage solid cancers, there were two partial responses $(4 \%, 2 / 51)$ seen in patients with FGFR2 non-amplified gastric cancer ${ }^{[42]}$.

In the SHINE study, a randomised phase II open label study of metastatic gastric cancer, 71 patients with FGFR2 amplification or polysomy were randomised to receive AZD4547 (amplified $n=18$, polysomy $n=$ 20; total $n=41$ ) or paclitaxel (amplified $n=15$, polysomy $n=15, n=30$ ). The median PFS was 1.8 months in the AZD4547 arm and 3.5 months in the paclitaxel arm (HR 1.57, 80\%CI: 1.12-2.21). The ORR was $2.6 \%$ in the AZD4547 arm and 23.3\% in the paclitaxel arm. The median OS was 5.5 and 6.6 months for the AZD4547 and paclitaxel arms, respectively (HR 1.31, 80\%CI: 0.89-1.95) ${ }^{[32]}$. Further work is required to identify why most patients with FGFR amplification have de novo resistance to this drug class and represents opportunities for clinical translational research and development of alternate strategies such as FGFR selective antibodies ${ }^{[43]}$.

\section{CLINICAL TRIALS IN SQUAMOUS LUNG CANCER}

In a phase Ib study in FGFR1 amplified squamous cell lung cancer, 15 patients received AZD4547. There was one partial response $(8 \%, 1 / 13)^{[44]}$ and the median overall survival was 4.9 months ${ }^{[44]}$. In a phase I study of infigratinib, amongst the 27 patients with squamous cell lung cancer harbouring a FGFR1 amplification, there were 4 partial responses $(8 \%)^{[31]}$. In the phase II LungMAP umbrella trial, there was only one objective response amongst 25 patients with evaluable disease with FGFR amplified or mutated squamous cell lung cancer $^{[30]}$.

\section{CLINICAL TRIALS IN BREAST CANCER}

In a phase II cohort of patients with HER2-negative breast cancer with FGFR1 amplification, 8 patients received AZD4547. There was one partial response $(13 \%, 1 / 8)^{[41]}$. In a phase I study of erdafitinib, none of the six patients with FGFR1 amplification achieved an objective response ${ }^{[45]}$. Similarly, no objective responses $(0 / 31)$ were observed in a phase I study of infigratinib in breast cancer $(F G F R 1 / 2 \text {, amplified } n=25)^{[31]}$. The lack of observed efficacy may be due to inadequate patient selection, ineffective compounds or the lack of oncogenic addiction of tumors harboring FGFR1 amplifications. Co-amplification of other genes within the FGFR1 containing 8p11-12 locus such as ZNF708 may act as an oncogenic driver ${ }^{[46]}$. FGFR1 amplifications are also associated with amplification of the 11q13 locus which contains putative oncogenes such as CCND1 and $F G F 19^{[47]}$.

\section{CLINICAL TRIALS IN OTHER TUMOUR TYPES}

In a phase II basket trial recruiting patients with FGFR and FGF ligand alterations, responses were observed in ovarian $(n=2)$, head and neck $(n=4)$, and primary CNS cancer $(n=1)$ with infigratinib ${ }^{[48]}$. In a phase I study of erdafinitib, responses were observed in a patient with endometrial cancer (FGFR2 fusion) and glioblastoma (FGFR3 fusion) $)^{[45]}$. These studies suggest that basket studies that recruit patients based on molecular characterisation represent a potential avenue to demonstration of drug activity and registration, especially in the context of the increasing use of next generation gene sequencing and profiling. 


\section{MECHANISMS OF ACQUIRED RESISTANCE}

Many pre-clinical studies have been conducted and are in progress to identify mechanisms of resistance to FGFR inhibitors that may contribute to poor clinical trial performance. Most of this work has been performed on urothelial, lung and gastric cancer cell lines which may not accurately reflect mechanisms of resistance in other tumour types (i.e., cholangiocarcinoma). Additionally these in vitro cell lines have a high degree of clonality and may not recapitulate the heterogeneity of human disease. Many of these mechanisms have not been validated in the clinical arena, highlighting the importance of post-progression tumour sampling and other novel strategies for detecting resistance mechanisms, such as plasma ctDNA monitoring for emergent mutations. Urine cfDNA is another promising modality for liquid biopsy in urothelial carcinoma ${ }^{[49]}$.

Despite limitations these pre-clinical studies involving models FGFR aberrations have demonstrated multiple mechanisms of acquired resistance to FGFR inhibitors which will be reviewed here. These include bypass signaling, epithelial to mesenchymal transition (EMT) and the emergence of secondary mutations in FGFR known as gatekeeper mutations. These studies have identified potential therapeutic strategies that can enhance the modest clinical benefit of FGFR targeting to date.

\section{ACTIVATION OF ALTERNATE RECEPTOR TYROSINE KINASES}

Resistance through bypass signaling occurs through the loss or switch of dependence of FGFR to other receptor tyrosine kinases, such as $\mathrm{MET}^{[50,51]}, \mathrm{Eph}_{3 \mathrm{~B}^{[52]}}, \mathrm{ERBB}_{2} / 3^{[53]}$ or $\mathrm{EGFR}^{[29]}$ [Figure 1].

Upregulation of the receptor tyrosine kinase MET has been described in DMS114 lung cancer cells (FGFR1 amplified), made resistant to infigratinib ${ }^{[50]}$ and H1581 lung cancer cells (FGFR1 amplified) made resistant to AZD4547 and the FGFR inhibitor rogaratinib ${ }^{[51]}$. In DMS114 resistant cells, transcriptional upregulation of MET led to reactivation of the MAPK signaling pathway. Similarly, in H1581 resistant cells, the elevated levels of MET led to increased downstream signaling pathways which were found to activate signaling in a ERBB3 dependent manner in cells resistant to AZD4547 and an ERBB3 independent manner in cells resistant to BAY $1163877^{[51]}$. The increased phosphorylation of the Ephrin 3B (Eph3B) receptor was found to be associated with acquired FGFR resistance in SNU-16 (FGFR2 amplified) gastric cancer cells, which could be reversed with small molecule inhibitors of Eph3 $\mathrm{B}^{[52]}$.

Switch to ERBB3 dependency has been further demonstrated in RT112 urothelial cancer cells (FGFR3-TACC3 fusion) following chronic exposure to infigratinib through upregulation of ERBB2/3 ligands ${ }^{[53]}$. Interestingly, upregulation of ERBB ligands only mediated resistance in FGFR3 dependent cells and not FGFR1 or FGFR2 dependent lines suggesting mechanisms of resistance may differ according to the FGFR alteration and cancer type ${ }^{[53]}$. EGFR has been shown to be a mediator of both acute and acquired resistance in FGFR3 mutant or fusion cell lines ${ }^{[29]}$. The sensitivity of FGFR inhibitors in FGFR3 dysregulated cell lines is largely mediated by intrinsic activation of EGFR. Acquired resistance in FGFR3 dependent cell lines occurs as EGFR is upregulated upon inhibition of FGFR through the release of negative feedback mechanisms partially compensating for the loss of FGFR signaling. Alternatively, intrinsic resistance to FGFR inhibition in FGFR3 mutant cells is due to EGFR dependency despite the presence of FGFR3 activating mutations, whereby EGFR is able to repress FGFR3 expression ${ }^{[29]}$.

Using a kinome-wide CRISPR/Cas9 screen, 20 kinases involving ILK (Integrin-linked kinase), SRC, and EGFR signaling were found to alter sensitivity to FGFR inhibition in FGFR2 amplified gastric cancer cell lines. Furthermore, in FGFR2 ${ }^{[54]}$ and FGFR $3^{[29]}$ dependent cell lines, co-targeting the FGFR inhibitor and EGFR or ERBB2/3 enhances anti-proliferative effects. 
A. Activation of alternate receptor tyrosine kinases and acquired gatekeeper mutations

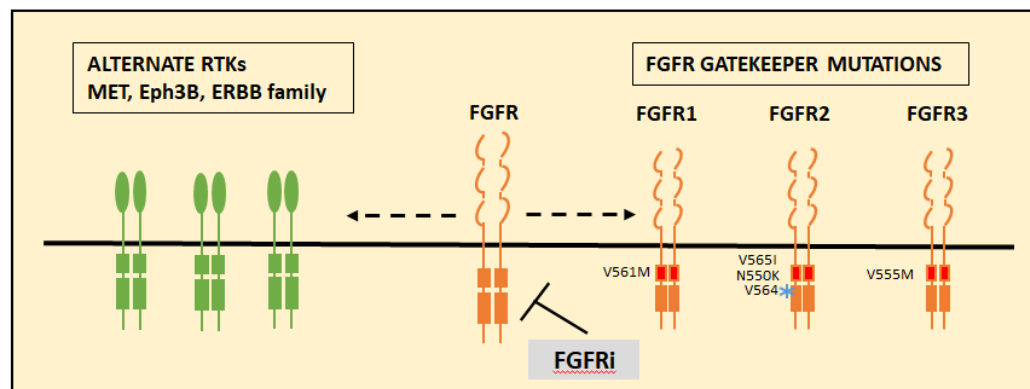

C. Activation of intracellular signaling pathways

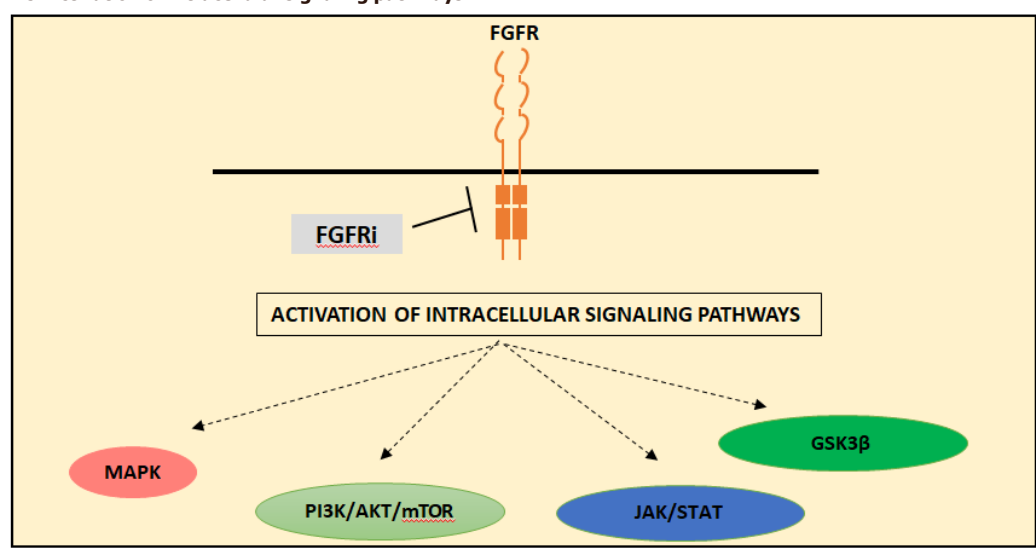

B. Epithelial to mesenchymal transition

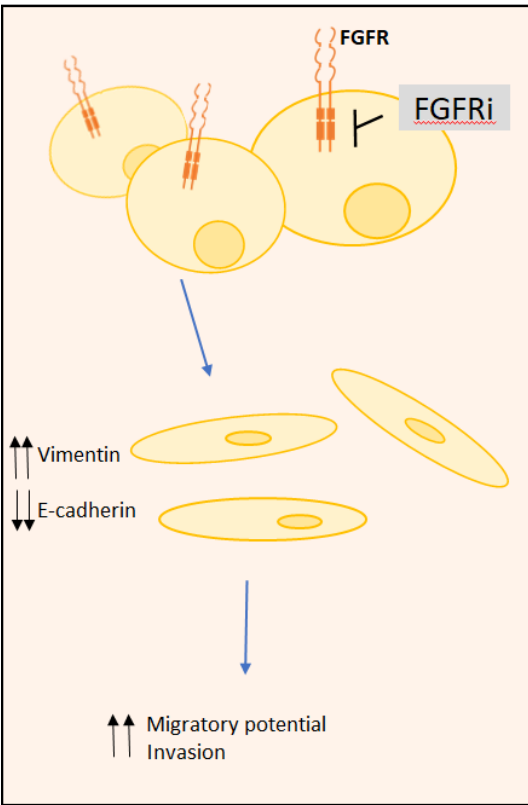

Figure 1. Mechanisms of acquired resistance to FGFR inhibition. Mechanisms of acquired resistance to FGFR inhibition can occur through activation of alternate receptor tyrosine kinases and acquired gatekeeper mutations (A), epithelial to mesenchymal transition (B) or activation of intracellular signaling pathways (C). FGFR: fibroblast growth factor receptor; FGFRi: fibroblast growth factor receptor inhibitor; RTK: receptor tyrosine kinase; MAPK: mitogen activation protein kinase; JAK/STAT: Janus kinase/signal transducers and activators of transcription; GSK3 $\beta$ : glycogen synthase kinase 3 beta

\section{ACTIVATION OF INTRACELLULAR SIGNALING PATHWAYS}

Bypass signaling can also occur due to changes within the PI3K/AKT/mTOR ${ }^{[55-57]}$, MAPK $^{[50,58,59]}$, STAT3 ${ }^{[60]}$ and GSK $3 \beta^{[61]}$ signaling pathways. Increased PI3K/AKT/mTOR signaling, independent of changes in upstream receptor tyrosine kinases has been described in DMS114 lung cancer cells (FGFR1 amplified) and RT112 urothelial cancer cells (FGFR3-TACC3) following chronic treatment with infigratinib ${ }^{[56]}$. Using deep sequencing, the dependency of PI3K/AKT/mTOR signaling in resistant DMS114 cells were found to be mediated by the emergence of an $A K T 1$ mutation ${ }^{[57]}$. In murine cell lines of stem cell leukemia syndrome containing FGFR1 amplification and made resistant to the non-selective FGFR inhibitor ponatinib, resistance was mediated by increased $\mathrm{PI} 3 \mathrm{~K} / \mathrm{AKT} / \mathrm{mTOR}$ signaling due to mutational inactivation of PTEN, a negative regulator of the pathway ${ }^{[55]}$.

Reactivation of PI3K/AKT/mTOR also occurs by of Pleckstrin Homology-Like Domain, family A, member 1 (PLHDA1) expression, a negative downregulator of $\mathrm{PIP}_{3} / \mathrm{AKT}$ binding. This effect has been observed in FGFR2 driven endometrial cancer cell lines with acquired resistance to FGFR inhibition and ERBB2 amplified breast cancer cells treated with anti-HER2 therapy suggested PLHDA1 may be a common resistance mechanism in RTK driven cancers ${ }^{[62]}$. Synergistic anti-tumour responses have been observed by co-targeting the FGFR and PI3K/AKT/mTOR pathway with PI3K ${ }^{[63]}$, mTOR $^{[64,65]}$ and Akt inhibitors ${ }^{[56,57,66]}$. The latter combination may be more effective in tumours harbouring a PIK3CA or PIK3R1 mutation ${ }^{[66]}$.

Constitutive activation of the MAPK signaling pathway has been shown to mediate FGFR resistance in both FGFR1 and FGFR2 amplified cell lines, however through different mechanisms. MAPK activation in FGFR1 amplified lines is shown to be mediated by a secondary mutation in NRAS ${ }^{[59]}$ and through 
chromosomal arm loss on chromosome 12p resulting in downregulation of DUSP $6^{[50]}$ a negative regulator of the MAPK pathway. Alternatively, in FGFR2 amplified cells constitutive MAPK signaling was mediated through the emergence of the BRAF fusion kinase JHM1D-BRAF which is demonstrated to enhance the dimerization capacity of BRAF ${ }^{[58]}$.

STAT3 has also been implicated in mediating resistance to AZD4547 and infigratinib in H1581 lung cancer cells (FGFR1 amplified) following induction of cognate receptors by the secretome. Acquired resistance was found to be overcome Hsp90 and HDAC inhibitors ${ }^{[60]}$. Independent phosphorylation and inactivation of GSK $3 \beta$ has been demonstrated as a mechanism of resistance to PDX models of FGFR 2 amplified diffuse gastric cancer made resistant through chronic treatment with AZD4547 ${ }^{[61]}$. Co-targetting of FGFR and ILK, an upstream receptor of GSK3 $\beta$ in FGFR2 amplified gastric cancer cell lines, resulted in synergistic antitumour responses ${ }^{[54]}$.

\section{EPITHELIAL-MESENCHYMAL TRANSITION}

The emergence of EMT following chronic exposure to FGFR inhibitors has also been described as a mechanism of resistance ${ }^{[6]]}$. Morphological changes defined by cells becoming more spindle shaped were observed in the gastric cancer cell line SNU-16 (FGFR2 amplification), following chronic exposure to AZD4547, infigratinib or PD173074. Consistent with changes in EMT, resistant cells displayed upregulation of vimentin and downregulation of the epithelial marker E-cadherin ${ }^{[52,6]}$. Furthermore, morphological changes in FGFR inhibitor resistant cell lines have also been reported in multiple cell lines including; RT112 resistant urothelial (FGFR3-TACC 3) ${ }^{[53,56]}$ and H1581 lung (FGFR1 amplified) ${ }^{[51]}$. In addition to morphological changes, H1581 resistant cells also showed enhanced migratory potential and invasion through matrigel, consistent with an EMT phenotype ${ }^{[53]}$.

\section{GATEKEEPER MUTATIONS}

Gatekeeper mutations, that modify the binding pocket to prevent drug binding, including FGFR1 ${\mathrm{V} 561 \mathrm{M}^{[55,68,69]} \text {, FGFR2 V565I/N550K/V564 } 4^{[70,71]} \text { and FGFR3 V555M }}^{[72]}$ are a distinct mechanism of resistance to FGFR inhibitors. Importantly, this mechanism implies tumours maintain their dependence on FGFR signaling and downstream pathway activation ${ }^{[73]}$. The FGFR1 V561 gatekeeper mutation is able to confer resistance though the stabilisation of the hydrophobic spine that favours the active conformation of FGFR1, which increases its autophosphorylation capacity ${ }^{[69]}$. Interestingly, the V561 mutation was shown to decrease the binding affinity to FGFR-VEGF inhibitor lucitanib by 500 fold whilst largely retaining binding affinity to the pan-FGFR inhibitor AZD4547 ${ }^{[69]}$. Resistance to AZD4547 by the V561 mutation is mediated by increased phosphorylation of STAT3 ${ }^{[68]}$. FGFR2 gatekeeper mutations V565I and N550K have been verified in $\mathrm{BaF} 3$ cells overexpressing FGFR2 and made resistant to dovitinib with both mutations working through different molecular mechanisms ${ }^{[70]}$. The V565I mutation confers resistance through steric hindrance of the drug into the ATP binding cleft whereas the N550K mutation confers resistance through stabilisation of FGFR2 into the active conformation ${ }^{[70]}$. The FGFR3 V555M mutation was identified in KMS-11 multiple myeloma cells (FGFR3 Y373C) made resistant to the FGFR inhibitor AZ12908010 ${ }^{[72]}$. Modelling of This mutation suggests amino acid substitution of valine to the methionine (with a larger side chain) would restrict access to the cavity adjacent to the ATP binding region ${ }^{[72]}$.

Using serial analysis of plasma cell-free DNA (cfDNA) ${ }^{[74]}$ isolated from three patients with FGFR2 fusion positive cholangiocarcinoma, receiving infigratinib, Goyal et al.$^{[7]}$ detected the emergence of resistance gatekeeper conferring mutations in FGFR2. Mutations at V564F, within the kinase domain developed in all three patients, suggesting this is common mechanism of resistance. Structural modelling suggested that this mutation caused a steric clash with infigratinib in the FGFR2 binding pocket. In two patients, multiple point mutations were detected (including V564F, N549H N549K, E565A, K659M, L617V) which 
are predicted to confer FGFR resistance. Conversely, PI3K pathway mutations were also detected in post progression and autopsy biopsies confirming the heterogeneity of these mutations underpins the complexity in treating these patients ${ }^{[71]}$. In urothelial carcinoma, the gatekeeper mutations FGFR3 V443L, $\mathrm{V} 443 \mathrm{M}$, and $\mathrm{L} 496 \mathrm{~V}$, were detected in cfDNA in 3 of 50 patients receiving erdafitnib ${ }^{[39]}$. Whilst not a gatekeeper mutation, a case report of a patient with FGFR2 amplified gastric cancer who responded to LY2874455, reported a resistance conferring FGFR2-ASCL5 fusion gene on post-progression biopsy ${ }^{[75]}$.

\section{STRATEGIES TO OVERCOME ACQUIRED FGFR RESISTANCE}

\section{Novel FGFR therapies}

Development of covalently binding specific FGFR inhibitors an active area of investigation as a therapeutic strategy to overcome resistance facilitated by gatekeeper mutations in the ATP binding pocket of FGFR ${ }^{[76]}$. Importantly, this strategy has potential to increase the duration of response as observed in EGFR-mutated lung cancer ${ }^{[77]}$ and represents an opportunity for development structurally optimised inhibitors. The FGFR inhibitor UPR1376, a chloroacetamide derivative has demonstrated preclinical anti-tumour activity in FGFR1 amplified lung cancer cell lines with acquired resistance to infigratinib ${ }^{[7]}$. Preliminary clinical results showed acquired resistance to infigratinib or Debio 1347 could be overcome with the covalently binding FGFR inhibitor TAS-120 in four patients with gatekeeper mutations ${ }^{[73]}$.

Monoclonal antibodies targeting FGFR have the ability to exert anti-tumour effects through antibodydependent cell-mediated cytotoxicity ${ }^{[79]}$. Bemarituzumab (FPA144, Five Prime Therapeutics) targeting the FGFR2b isoform is currently in clinical testing (NCT03694522) in gastric carcinoma. FGF traps which sequester FGF ligands and prevent binding of ligands to FGFRs have been proposed as a therapeutic strategy, however its utility in FGFR mutational driven tumours is uncertain ${ }^{[80]}$. GSK3052230, a novel engineered FGF trap comprised of the extracellular domain of FGFR1 fused to the Fc portion and is in clinical testing in lung carcinoma (NCT01868022).

\section{Combination strategies}

Clinical trials are exploring the strategy of upfront combination therapy to forestall the development of acquired resistance to selective FGFR inhibitors. In a phase I trial combination therapies of FGFR and PI3K inhibitors (infigratinib/BYL719) were tested in tumours with PIK3CA mutations or genetic alterations in FGFR1-3. Objective responses were observed in urothelial, head and neck, melanoma and anal cancer, however it is unclear whether this this combination is any more effective over single agent therapy ${ }^{[8]}$.

The combination of FGFR and immune checkpoint inhibitors are supported by marked anti-tumour activity in mouse models ${ }^{[82]}$. As checkpoint inhibition has been proven to be efficacious in refractory urothelial carcinoma ${ }^{[33-85]}$, the monoclonal anti-FGFR3 inhibitor B-701 is currently in clinical testing with the PD-1 antibody pembrolizumab (NCT03123055). Other immune checkpoint combinations in clinical testing in urothelial carcinoma include erdafitinib with JNJ-63723283 (NCT03473743), rogaratinib with atezolizumab (NCT03473756) and AZD4547 with durvalumab (NCT02546661).

\section{CONCLUSION}

The mechanisms of FGFR resistance are diverse and include the activation of alternate receptor tyrosine kinases, induction of alternate cellular signaling pathways, induction of EMT and emergence of gatekeeper mutations. Further preclinical and translational clinical studies are paramount to define the mechanisms of resistance and design more rational treatments to overcome drug resistance. Combination treatment strategies to overcome bypass signaling and next generation FGFR inhibitors to circumvent gatekeeper mutations are promising avenues to improve the clinical use of FGFR inhibitors. 


\section{DECLARATIONS}

\section{Authors' contributions}

Made substantial contributions to conception and design of the study, performed data analysis and interpretation, performed data acquisition, as well as provided administrative, technical, and material support: Lau DK, Jenkins L, Weickhardt A

\section{Availability of data and materials}

Not applicable.

\section{Financial support and sponsorship}

Lau DK received financial support from La Trobe University (Australian Postgraduate Award), Pancare/ RMA Network; Jenkins L received financial support from La Trobe University (Australian Postgraduate Award); Weickhardt A has received consulting fees from Merck, Novartis, Pfizer and Ipsen.

\section{Conflicts of interest}

\section{Weickhardt A}

Research funding: Bristol Myers Squibb; Novartis, Pfizer

Advisory board: Bristol Myers Squibb; Novartis; Pfizer; Merck

Honoraria: Merck/MSD

David Lau/Laura Jenkins: Nil

\section{Ethical approval and consent to participate}

Not applicable.

\section{Consent for publication}

Not applicable.

\section{Copyright}

(c) The Author(s) 2019.

\section{REFERENCES}

1. Brooks AN, Kilgour E, Smith PD. Molecular pathways: fibroblast growth factor signaling: a new therapeutic opportunity in cancer. Clin Cancer Res 2012;18:1855-62.

2. Babina IS, Turner NC. Advances and challenges in targeting FGFR signalling in cancer. Nat Rev Cancer 2017;17:318-32.

3. Dienstmann R, Patnaik A, Garcia-Carbonero R, Cervantes A, Benavent M, et al. Safety and activity of the first-in-class Sym004 antiEGFR antibody mixture in patients with refractory colorectal cancer. Cancer Discov 2015;5:598-609.

4. Hall TG, Yu Y, Eathiraj S, Wang Y, Savage RE, et al. Preclinical activity of ARQ 087, a novel inhibitor targeting FGFR dysregulation. PLoS One 2016;11:e0162594.

5. Gavine PR, Mooney L, Kilgour E, Thomas AP, Al-Kadhimi K, et al. AZD4547: an orally bioavailable, potent, and selective inhibitor of the fibroblast growth factor receptor tyrosine kinase family. Cancer Res 2012;72:2045-56.

6. Guagnano V, Kauffmann A, Wöhrle S, Stamm C, Ito M, et al. FGFR genetic alterations predict for sensitivity to NVP-BGJ398, a selective Pan-FGFR inhibitor. Cancer Discov 2012;2:1118-33.

7. Perera TPS, Jovcheva E, Mevellec L, Vialard J, De Lange D, et al. Discovery and pharmacological characterization of JNJ-42756493 (Erdafitinib), a functionally selective small-molecule FGFR family inhibitor. Mol Cancer Ther 2017;16:1010-20.

8. Kalyukina M, Yosaatmadja Y, Middleditch MJ, Patterson AV, Smaill JB, et al. TAS-120 cancer target binding: defining reactivity and revealing the first fibroblast growth factor receptor 1 (FGFR1) irreversible structure. ChemMedChem 2019;14:494-500.

9. Hollebecque A, Lihou C, Zhen H, Abou-Alfa GK, Borad M, et al. 756PInterim results of fight-202, a phase II, open-label, multicenter study of INCB054828 in patients (pts) with previously treated advanced/metastatic or surgically unresectable cholangiocarcinoma (CCA) with/without fibroblast growth factor (FGF)/FGF receptor (FGFR) genetic alterations. Ann Oncol 2018;29.

10. Murtuza A, Bulbul A, Shen JP, Keshavarzian P, Woodward BD, et al. Novel third-generation EGFR tyrosine kinase inhibitors and strategies to overcome therapeutic resistance in lung cancer. Cancer Res 2019;79:689-98.

11. McCoach CE, Le AT, Gowan K, Jones K, Schubert L, et al. Resistance mechanisms to targeted therapies in ROS1(+) and ALK $(+)$ non- 
small cell lung cancer. Clin Cancer Res 2018;24:3334-47.

12. McLean SR, Gana-Weisz M, Hartzoulakis B, Frow R, Whelan J, et al. Imatinib binding and cKIT inhibition is abrogated by the cKIT kinase domain I missense mutation Val654Ala. Mol Cancer Ther 2005;4:2008-15.

13. Wu YM, Su F, Kalyana-Sundaram S, Khazanov N, Ateeq B, et al. Identification of targetable FGFR gene fusions in diverse cancers. Cancer Discov 2013;3:636-47.

14. Wang Y, Ding X, Wang S, Moser CD, Shaleh HM, et al. Antitumor effect of FGFR inhibitors on a novel cholangiocarcinoma patient derived xenograft mouse model endogenously expressing an FGFR2-CCDC6 fusion protein. Cancer Lett 2016;380:163-73.

15. Sia D, Losic B, Moeini A, Cabellos L, Hao K, et al. Massive parallel sequencing uncovers actionable FGFR2-PPHLN1 fusion and ARAF mutations in intrahepatic cholangiocarcinoma. Nat Commun 2015;6:6087.

16. Arai Y, Totoki Y, Hosoda F, Shirota T, Hama N, et al. Fibroblast growth factor receptor 2 tyrosine kinase fusions define a unique molecular subtype of cholangiocarcinoma. Hepatology 2014;59:1427-34.

17. Nakamura H, Arai Y, Totoki Y, Shirota T, Elzawahry A, et al. Genomic spectra of biliary tract cancer. Nat Genet 2015;47:1003-10.

18. Bakkar AA, Wallerand H, Radvanyi F, Lahaye JB, Pissard S, et al. FGFR3 and TP53 gene mutations define two distinct pathways in urothelial cell carcinoma of the bladder. Cancer Res 2003;63:8108-12.

19. Lamy A, Gobet F, Laurent M, Blanchard F, Varin C, et al. Molecular profiling of bladder tumors based on the detection of FGFR3 and TP53 mutations. J Urol 2006;176:2686-9.

20. Williams SV, Hurst CD, Knowles MA. Oncogenic FGFR3 gene fusions in bladder cancer. Hum Mol Genet 2013;22:795-803.

21. Byron SA, Gartside M, Powell MA, Wellens CL, Gao F, et al. FGFR2 point mutations in 466 endometrioid endometrial tumors: relationship with MSI, KRAS, PIK3CA, CTNNB1 mutations and clinicopathological features. PLoS one 2012;7:e30801.

22. Weiss J, Sos ML, Seidel D, Peifer M, Zander T, et al. Frequent and focal FGFR1 amplification associates with therapeutically tractable FGFR1 dependency in squamous cell lung cancer. Science translational medicine 2010;2:62ra93.

23. Heist RS, Mino-Kenudson M, Sequist LV, Tammireddy S, Morrissey L, et al. FGFR1 amplification in squamous cell carcinoma of the lung. J Thorac Oncol 2012;7:1775-80.

24. Helsten T, Elkin S, Arthur E, Tomson BN, Carter J, et al. The FGFR landscape in cancer: analysis of 4,853 tumors by next-generation sequencing. Clin Cancer Res 2016;22:259-67.

25. Turner N, Lambros MB, Horlings HM, Pearson A, Sharpe R, et al. Integrative molecular profiling of triple negative breast cancers identifies amplicon drivers and potential therapeutic targets. Oncogene 2010;29:2013-23.

26. Su X, Zhan P, Gavine PR, Morgan S, Womack C, et al. FGFR2 amplification has prognostic significance in gastric cancer: results from a large international multicentre study. Br J Cancer 2014;110:967-75.

27. Guagnano V, Kauffmann A, Wohrle S, Stamm C, Ito M, et al. FGFR genetic alterations predict for sensitivity to NVP-BGJ398, a selective pan-FGFR inhibitor. Cancer Discov 2012;2:1118-33.

28. Karkera JD, Cardona GM, Bell K, Gaffney D, Portale JC, et al. Oncogenic characterization and pharmacologic sensitivity of activating fibroblast growth factor receptor (FGFR) genetic alterations to the selective FGFR inhibitor Erdafitinib. Mol Cancer Ther 2017;16:1717-26.

29. Herrera-Abreu MT, Pearson A, Campbell J, Shnyder SD, Knowles MA, et al. Parallel RNA interference screens identify EGFR activation as an escape mechanism in FGFR3-mutant cancer. Cancer Discov 2013;3:1058-71.

30. Aggarwal C, Redman MW, Lara P, Borghaei H, Hoffman PC, et al. Phase II study of the FGFR inhibitor AZD4547 in previously treated patients with FGF pathway-activated stage IV squamous cell lung cancer (SqNSCLC): LUNG-MAP sub-study SWOG S1400D. J Clin Oncol 2017;35:9055.

31. Nogova L, Sequist LV, Garcia JMP, Andre F, Delord JP, et al. Evaluation of BGJ398, a fibroblast growth factor receptor 1-3 kinase inhibitor, in patients with advanced solid tumors harboring genetic alterations in fibroblast growth factor receptors: results of a global phase I, dose-escalation and dose-expansion study. J Clin Oncol 2017;35:157-65.

32. Van Cutsem E, Bang YJ, Mansoor W, Petty RD, Chao Y, et al. A randomized, open-label study of the efficacy and safety of AZD4547 monotherapy versus paclitaxel for the treatment of advanced gastric adenocarcinoma with FGFR2 polysomy or gene amplification. Ann Oncol 2017;28:1316-24.

33. Javle M, Lowery M, Shroff RT, Weiss KH, Springfeld C, et al. Phase II study of BGJ398 in patients with FGFR-altered advanced cholangiocarcinoma. J Clin Oncol 2018;36:276-82.

34. Mazzaferro V, El-Rayes BF, Droz Dit Busset M, Cotsoglou C, Harris WP, et al. Derazantinib (ARQ 087) in advanced or inoperable FGFR2 gene fusion-positive intrahepatic cholangiocarcinoma. Br J Cancer 2019;120:165-71.

35. Bahleda R, Italiano A, Hierro C, Mita AC, Cervantes A, et al. Multicenter Phase I Study of Erdafitinib (JNJ-42756493), Oral PanFibroblast Growth Factor Receptor Inhibitor, in Patients with Advanced or Refractory Solid Tumors. Clin Cancer Res 2019; doi: 10.1158/1078-0432

36. Chen YY, Qing M, Li J, De Porre P, Park JO, et al. 624PDPreliminary results of a ph2a study to evaluate the clinical efficacy and safety of erdafitinib in Asian patients with biomarker-selected advanced cholangiocarcinoma (CCA). Ann Oncol 2018;29.

37. Meric-Bernstam F, He H, Huang J, Winkler R, Arkenau H, et al. O-001Efficacy of TAS-120, an irreversible fibroblast growth factor receptor (FGFR) inhibitor, in cholangiocarcinoma patients with FGFR pathway alterations who were previously treated with chemotherapy and other FGFR inhibitors. Ann Oncol 2018;29.

38. Siefker-Radtke AO, Necchi A, Park SH, GarcÃa-Donas JS, Huddart RA, et al. First results from the primary analysis population of the phase 2 study of erdafitinib (ERDA; JNJ-42756493) in patients (pts) with metastatic or unresectable urothelial carcinoma (mUC) and FGFR alterations (FGFRalt). J Clin Oncol 2018;36:4503. 
39. Pal SK, Rosenberg JE, Hoffman-Censits JH, Berger R, Quinn DI, et al. Efficacy of BGJ398, a fibroblast growth factor receptor 1-3 inhibitor, in patients with previously treated advanced urothelial carcinoma with FGFR3 alterations. Cancer Discov 2018;8:812-21.

40. Arkenau HT, Saggese M, Hollebecque A, Mathewson A, Lemech CR, et al. A phase 1 expansion cohort of the fibroblast growth factor receptor (FGFR) inhibitor AZD4547 in patients (pts) with advanced gastric (GC) and gastroesophageal (GOJ) cancer. J Clin Oncol 2014;32:2620.

41. Pearson A, Smyth E, Babina IS, Herrera-Abreu MT, Tarazona N, et al. High-level clonal FGFR amplification and response to FGFR inhibition in a translational clinical trial. Cancer Discov 2016;6:838-51.

42. Michael M, Bang YJ, Park YS, Kang YK, Kim TM, et al. A phase 1 study of LY2874455, an oral selective pan-FGFR inhibitor, in patients with advanced cancer. Target Oncol 2017;12:463-74.

43. Necchi A, Castellano DE, Mellado B, Pang S, Urun Y, et al. Fierce-21: phase II study of vofatmab (B-701), a selective inhibitor of FGFR3, as salvage therapy in metastatic urothelial carcinoma (mUC). J Clin Oncol 2019;37:409.

44. Paik PK, Shen R, Berger MF, Ferry D, Soria JC, et al. A phase Ib open-label multicenter study of AZD4547 in patients with advanced squamous cell lung cancers. Clin Cancer Res 2017;23:5366-73.

45. Tabernero J, Bahleda R, Dienstmann R, Infante JR, Mita A, et al. Phase I dose-escalation study of JNJ-42756493, an oral panfibroblast growth factor receptor inhibitor, in patients with advanced solid tumors. J Clin Oncol 2015;33:3401-8.

46. Kwek SS, Roy R, Zhou H, Climent J, Martinez-Climent JA, et al. Co-amplified genes at 8p12 and 11q13 in breast tumors cooperate with two major pathways in oncogenesis. Oncogene 2009;28:1892-903.

47. Letessier A, Sircoulomb F, Ginestier C, Cervera N, Monville F, et al. Frequency, prognostic impact, and subtype association of 8p12, 8q24, 11q13, 12p13, 17q12, and 20q13 amplifications in breast cancers. BMC Cancer 2006;6:245.

48. Slosberg ED, Kang BP, Peguero J, Taylor M, Bauer TM, et al. Signature program: a platform of basket trials. Oncotarget 2018;9:2138395.

49. Lee DH, Yoon H, Park S, Kim JS, Ahn YH, et al. Urinary exosomal and cell-free DNA detects somatic mutation and copy number alteration in urothelial carcinoma of bladder. Sci Rep 2018;8:14707.

50. Malchers F, Ercanoglu M, Schutte D, Castiglione R, Tischler V, et al. Mechanisms of primary drug resistance in FGFR1-amplified lung cancer. Clin Cancer Res 2017;23:5527-36.

51. Kim SM, Kim H, Yun MR, Kang HN, Pyo KH, et al. Activation of the Met kinase confers acquired drug resistance in FGFR-targeted lung cancer therapy. Oncogenesis 2016;5:e241.

52. Lee SY, Na YJ, Jeong YA, Kim JL, Oh SC, et al. Upregulation of EphB3 in gastric cancer with acquired resistance to a FGFR inhibitor. Int J Biochem Cell Biol 2018;102:128-37.

53. Wang J, Mikse O, Liao RG, Li Y, Tan L, et al. Ligand-associated ERBB2/3 activation confers acquired resistance to FGFR inhibition in FGFR3-dependent cancer cells. Oncogene 2015;34:2167-77.

54. Chen J, Bell J, Lau BT, Whittaker T, Stapleton D, et al. A functional CRISPR/Cas9 screen identifies kinases that modulate FGFR inhibitor response in gastric cancer. Oncogenesis 2019;8:33.

55. Cowell JK, Qin H, Hu T, Wu Q, Bhole A, et al. Mutation in the FGFR1 tyrosine kinase domain or inactivation of PTEN is associated with acquired resistance to FGFR inhibitors in FGFR1-driven leukemia/lymphomas. Int J Cancer 2017;141:1822-9.

56. Datta J, Damodaran S, Parks H, Ocrainiciuc C, Miya J, et al. Akt activation mediates acquired resistance to fibroblast growth factor receptor inhibitor BGJ398. Mol Cancer Ther 2017;16:614-24.

57. Gimenez-Xavier P, Pros E, Aza A, Moran S, Tonda R, et al. Deep analysis of acquired resistance to FGFR1 inhibitor identifies MET and AKT activation and an expansion of AKT1 mutant cells. Oncotarget 2018;9:31549-58.

58. Sase H, Nakanishi Y, Aida S, Horiguchi-Takei K, Akiyama N, et al. Acquired JHDM1D-BRAF fusion confers resistance to FGFR inhibition in FGFR2-amplified gastric cancer. Mol Cancer Ther 2018;17:2217-25.

59. Bockorny B, Rusan M, Chen W, Liao RG, Li Y, et al. RAS-MAPK reactivation facilitates acquired resistance in FGFR1-amplified lung cancer and underlies a rationale for upfront FGFR-MEK blockade. Mol Cancer Ther 2018;17:1526-39.

60. Wang X, Ai J, Liu H, Peng X, Chen H, et al. The secretome engages STAT3 to favor a cytokine-rich microenvironment in mediating acquired resistance to FGFR inhibitors. Mol Cancer Ther 2019;18:667-79.

61. Lau WM, Teng E, Huang KK, Tan JW, Das K, et al. Acquired resistance to FGFR inhibitor in diffuse-type gastric cancer through an AKT-independent PKC-mediated phosphorylation of GSK3beta. Mol Cancer Ther 2018;17:232-42.

62. Fearon AE, Carter EP, Clayton NS, Wilkes EH, Baker AM, et al. PHLDA1 mediates drug resistance in receptor tyrosine kinase-driven cancer. Cell Rep 2018;22:2469-81.

63. Wang L, Sustic T, Leite de Oliveira R, Lieftink C, Halonen P, et al. A functional genetic screen identifies the phosphoinositide 3-kinase pathway as a determinant of resistance to fibroblast growth factor receptor inhibitors in FGFR mutant urothelial cell carcinoma. Eur Urol 2017;71:858-62.

64. Gozgit JM, Squillace RM, Wongchenko MJ, Miller D, Wardwell S, et al. Combined targeting of FGFR2 and mTOR by ponatinib and ridaforolimus results in synergistic antitumor activity in FGFR2 mutant endometrial cancer models. Cancer Chemother Pharmacol 2013;71:1315-23.

65. Hu Y, Lu H, Zhang J, Chen J, Chai Z, et al. Essential role of AKT in tumor cells addicted to FGFR. Anticancer Drugs 2014;25:183-8.

66. Yu Y, Hall T, Eathiraj S, Wick MJ, Schwartz B, et al. In-vitro and in-vivo combined effect of ARQ 092, an AKT inhibitor, with ARQ 087, a FGFR inhibitor. Anticancer Drugs 2017;28:503-13.

67. Grygielewicz P, Dymek B, Bujak A, Gunerka P, Stanczak A, et al. Epithelial-mesenchymal transition confers resistance to selective FGFR inhibitors in SNU-16 gastric cancer cells. Gastric Cancer 2016;19:53-62. 
68. Ryan MR, Sohl CD, Luo B, Anderson KS. The FGFR1 V561M gatekeeper mutation drives AZD4547 resistance through STAT3 activation and EMT. Mol Cancer Res 2019;17:532-43.

69. Sohl CD, Ryan MR, Luo B, Frey KM, Anderson KS. Illuminating the molecular mechanisms of tyrosine kinase inhibitor resistance for the FGFR1 gatekeeper mutation: the Achilles' heel of targeted therapy. ACS Chem Biol 2015;10:1319-29.

70. Byron SA, Chen H, Wortmann A, Loch D, Gartside MG, et al. The N550K/H mutations in FGFR2 confer differential resistance to PD173074, dovitinib, and ponatinib ATP-competitive inhibitors. Neoplasia 2013;15:975-88.

71. Goyal L, Saha SK, Liu LY, Siravegna G, Leshchiner I, et al. Polyclonal secondary FGFR2 mutations drive acquired resistance to FGFR inhibition in patients with FGFR2 fusion-positive cholangiocarcinoma. Cancer Discov 2017;7:252-63.

72. Chell V, Balmanno K, Little AS, Wilson M, Andrews S, et al. Tumour cell responses to new fibroblast growth factor receptor tyrosine kinase inhibitors and identification of a gatekeeper mutation in FGFR3 as a mechanism of acquired resistance. Oncogene 2013;32:3059-70.

73. Goyal L, Shi L, Liu LY, Fece de la Cruz F, Lennerz JK, et al. TAS-120 overcomes resistance to ATP-competitive FGFR inhibitors in patients with FGFR2 fusion-positive intrahepatic cholangiocarcinoma. Cancer Discov 2019; doi: 10.1158/2159-8290.cd-19-0182.

74. Anker P, Stroun M. Circulating DNA in plasma or serum. Medicina (B Aires) 2000;60:699-702.

75. Kim SY, Ahn T, Bang H, Ham JS, Kim J, et al. Acquired resistance to LY2874455 in FGFR2-amplified gastric cancer through an emergence of novel FGFR2-ACSL5 fusion. Oncotarget 2017;8:15014-22.

76. Tan L, Wang J, Tanizaki J, Huang Z, Aref AR, et al. Development of covalent inhibitors that can overcome resistance to firstgeneration FGFR kinase inhibitors. Proc Natl Acad Sci U S A 2014;111:E4869-77.

77. Soria JC, Ohe Y, Vansteenkiste J, Reungwetwattana T, Chewaskulyong B, et al. Osimertinib in untreated EGFR-mutated advanced non-small-cell lung cancer. N Engl J Med 2017; doi: 10.1056/NEJMoa1713137.

78. Fumarola C, Bozza N, Castelli R, Ferlenghi F, Marseglia G, et al. Expanding the arsenal of FGFR inhibitors: a novel chloroacetamide derivative as a new irreversible agent with anti-proliferative activity against FGFR1-amplified lung cancer cell lines. Front Oncol 2019;9:179.

79. Bendell JC, Rogers S, Xiang H, Pierce KL, Krishnan K, et al. FPA144-001: a first in human study of FPA 144, an ADCC-enhanced, FGFR2b isoform-selective monoclonal antibody in patients with advanced solid tumors. J Clin Oncol 2016;34:140.

80. Presta M, Chiodelli P, Giacomini A, Rusnati M, Ronca R. Fibroblast growth factors (FGFs) in cancer: FGF traps as a new therapeutic approach. Pharmacol Ther 2017;179:171-87.

81. Hyman DM, Tran B, Corral Jaime J, Garralda E, Machiels JPH, et al. Phase Ib study of BGJ398 in combination with BYL719 in patients (pts) with select advanced solid tumors. J Clin Oncol 2016;34:2500.

82. Palakurthi S, Kuraguchi M, Zacharek S, Liu J, Bonal D, et al. Abstract B27: improved survival with erdafitinib (JNJ-42756493) and PD-1 blockade mediated by enhancement of anti-tumor immunity in an FGFR2-driven genetically engineered mouse model of lung cancer. Cancer Immunol Res 2017;5:B27.

83. Sharma P, Retz M, Siefker-Radtke A, Baron A, Necchi A, et al. Nivolumab in metastatic urothelial carcinoma after platinum therapy (CheckMate 275): a multicentre, single-arm, phase 2 trial. Lancet Oncol 2017;18:312-22.

84. Bellmunt J, De Wit R, Vaughn DJ, Fradet Y, Lee JL, et al. Pembrolizumab as second-line therapy for advanced urothelial carcinoma. N Engl J Med 2017;376:1015-26.

85. Patel MR, Ellerton J, Infante JR, Agrawal M, Gordon M, et al. Avelumab in metastatic urothelial carcinoma after platinum failure (JAVELIN Solid Tumor): pooled results from two expansion cohorts of an open-label, phase 1 trial. Lancet Oncol 2018;19:51-64.

86. Joerger M, Cassier P, Penel N, Cathomas R, Richly H, et al. Rogaratinib treatment of patients with advanced urothelial carcinomas prescreened for tumor FGFR mRNA expression. J Clin Oncol 2018;36:494.

87. Necchi A, Serbest G, Zhen H, Loriot Y, Pouessel D, et al. 900PInterim results of fight-201, a phase II, open-label, multicenter study of INCB054828 in patients (pts) with metastatic or surgically unresectable urothelial carcinoma (UC) harboring fibroblast growth factor (FGF)/FGF receptor (FGFR) genetic alterations (GA). Ann Oncol 2018;29. 